\title{
Analysis of solar narrow band dm-spikes observed at 1420 and $2695 \mathrm{MHz}$
}

\author{
H. Mészárosová ${ }^{1}$, A. Veronig ${ }^{2}$, P. Zlobec ${ }^{3}$, and M. Karlický1 \\ 1 Astronomical Institute of the Academy of Sciences of the Czech Republic, 25165 Ondřejov, Czech Republic \\ ${ }^{2}$ Institute for Geophysics, Astrophysics and Meteorology, University of Graz, Universitätsplatz 5, 8010 Graz, Austria \\ 3 INAF - Trieste Astronomical Observatory, Via G.B. Tiepolo 11, 34131 Trieste, Italy
}

Received 20 January 2003 / Accepted 2 June 2003

\begin{abstract}
Using both linear and nonlinear methods, narrow band dm-spikes recorded at 1420 and $2695 \mathrm{MHz}$ on June 6, 2000, July 8, 2000, July 12, 2000, July 20, 2000, and March 28, 2001 were analyzed. In particular their time profiles were studied statistically. The mean characteristic times of the ascending and of the decaying parts of their profiles are comparable, even if the dispersion of the values is very broad. For selected spikes at $1420 \mathrm{MHz}$ a more precise fitting technique using exponential profiles was applied. While in the decaying part the exponential trend can be generally found, in the ascending part the exponential form can be confirmed only in few cases. The ascending and decaying phase of spikes presumably correspond to the source instability evolution and the plasma wave absorption. Furthermore, durations and polarization values of both 1420 and $2695 \mathrm{MHz}$ spikes were determined and compared with the results in literature. All the analyzed spike events were located near the solar disk center. The polarization values and their trend in spike groups and the nearly constant duration suggest that the polarization originates at the source itself or near it. Selected time series of spikes were tested with respect to low-dimensional determinism and nonlinearity. We found that spikes recorded at fixed frequencies are not governed by a linear stochastic process, as the underlying physical system contains nonlinear signatures.
\end{abstract}

Key words. Sun: radio radiation - Sun: flares - methods: data analysis - methods: statistical

\section{Introduction}

Due to their exceptionally high brightness temperature $\left(T_{\mathrm{b}} \approx\right.$ $10^{15} \mathrm{~K}$ ) and short duration $(\leq 0.1 \mathrm{~s})$, narrowband spikes (see reviews by Benz 1986 and Fleishman \& Melnikov 1998) belong to one of the most interesting species of solar radio bursts. Their characteristics were described in several papers (e.g. Slottje 1981; Benz et al. 1982; Karlický 1984; Stähli \& Benz 1987).

Theoretical emission models can be divided into two groups that are based on: a) plasma emission and acceleration processes (Kuijpers et al. 1981; Tajima et al. 1990; Wentzel 1991; Bárta \& Karlický 2001); b) electron-cyclotron maser emission (Holman et al. 1980; Melrose \& Dulk 1982; Vlahos \& Sharma 1984; Winglee et al. 1988; Aschwanden 1990; Fleishman \& Yastrebov 1994; Fleishman \& Melnikov 1998). Furthermore, spikes were interpreted as radio emission of electrons accelerated in MHD cascading waves generated in plasma outflows from the magnetic reconnection region (Karlický et al. 1996). To distinguish between different models, various spike characteristics were studied, in particular polarization and harmonic structures (Güdel 1990; Güdel \& Zlobec 1991; Krucker \& Benz 1994). Duration and time profile

Send offprint requests to: $\mathrm{H}$. Mészárosová, e-mail: hana@asu.cas.cz are also important criteria for such a test. An important study was carried out by Güdel \& Benz (1990) who considered duration, decay phase and exciter function within a broad frequency range (from 362 to $1010 \mathrm{MHz}$ ). In particular, they determined the e-folding decay time $\tau$ and the spike duration $d_{\mathrm{e}}$ (natural duration) as function of frequency:

$\tau(v)=0.0165\left(\frac{v}{661}\right)^{-1.06} \mathrm{~s}$

$d_{\mathrm{e}}(v) \approx 0.0348\left(\frac{v}{661}\right)^{-1.34} \mathrm{~s}$,

where $v$ is the radio frequency in MHz.

In this paper, we present some spike characteristics at 1420 and $2695 \mathrm{MHz}: 1)$ detection of the e-folding decay time using statistical methods as well as detailed analysis of selected spikes; 2) evaluation of duration, polarization and time delay between the two polarimetric components; 3) search of possible low-dimensional determinism and nonlinearity, using fractal dimensions and surrogate data techniques.

Fractal dimension methods had already been applied to spike time series by Isliker (1992), Isliker \& Benz (1994), Mészárosová et al. (2000) and Veronig et al. (2000). In these papers no indication for low-dimensional determinism was found. In the present study we used high-time-resolution 
observations ( $1 \mathrm{~ms}$ ). Such a high digitization rate is fundamental for the analysis of very rapidly evolving structures. In particular, insufficient time resolution may obscure the signatures of low-dimensional determinism in the analyzed time series. Using a $1 \mathrm{~ms}$ temporal resolution, we revisit the determination of fractal dimensions of narrowband spikes. Furthermore, we tested whether nonlinearity is present in the data using the method of surrogate time series. The surrogate data test is a type of nonlinear technique softer than the fractal dimension determination, as it checks for the presence of nonlinearity in the data without presuming the existence of determinism.

\section{Observations}

The observations we considered were recorded simultaneously by the Trieste Astronomical Observatory radiopolarimeter and the Ondřejov radiospectrograph. Only high-time-resolution observations (digitization rate $1000 \mathrm{~Hz}$ ) recorded at 1420 and $2695 \mathrm{MHz}$ were analyzed. The spectrographic observations were used in order to identify the presence of spikes and their extension in the frequency domain.

In Table 1 we list six intervals that were selected for the different types of analyses together with the associated optical and soft X-ray flares. It is interesting that most of the flares associated with spikes were relatively weak, just subflares, and that they all were located near the disk center (Solar Geophysical Data bulletin).

On the other hand, the spikes recorded on June 6, 2000 were associated with a huge flare of 3B optical importance localized at N20 E18 in AR 9026. The spikes were evolving during the decaying phase of the flare, which was of long duration and high importance (similar to the June 15, 1991 event described in Zlobec \& Karlický 1998). Contrary to this case, the other spike events considered developed during the rising phase of the associated flares, which were predominantly of low importance (see Table 1).

\section{Linear analysis}

\subsection{Spike time profiles}

Important information about spikes is contained in their time profile. For the determination of the e-folding rise and decay times $\left(t_{\mathrm{a}}\right.$ and $\left.t_{\mathrm{d}}\right)$ four intervals were selected (Tables 2 and 3). During that time about 10000 spikes were identified with different occurrence rate and different polarization degree. For the statistical approach the full set of spikes listed in Table 2 was used.

In order to determine the possible exponential trend detailed analysis was performed using a more precise fitting method for a limited number of selected spikes (Table 4). It is assumed that the ascending and the decaying part correspond essentially to the source instability evolution and to the plasma waves absorption, respectively.

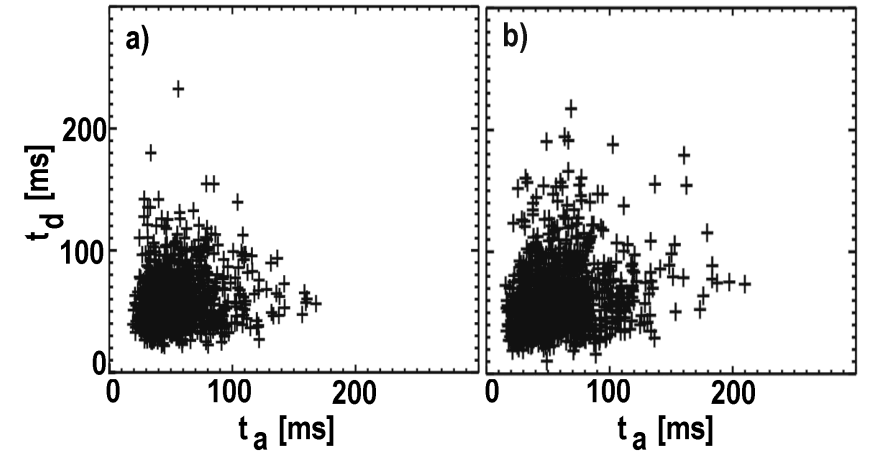

Fig. 1. Scatter plots of the characteristic times ( $t_{\mathrm{d}}$ versus $\left.t_{\mathrm{a}}\right)$ : a) July $08,2000 R$-components of the spikes at $1420 \mathrm{MHz}$, b) March 28, $2001 R$-components of the spikes at $2695 \mathrm{MHz}$.

\subsubsection{Statistical study}

The observing frequency, the maximum and the minimum flux density values and the number of the considered spikes in the corresponding intervals are given in Table 2. In general, the analysis was performed separately for the left (L) and the right $(\mathrm{R})$ polarimetric channel (see " $\mathrm{L}$ " and " $\mathrm{R}$ " in Table 2 and 3). The polarization of the July 12, 2000 and July 20, 2000 spikes was almost zero, therefore only data from one (L) channel were considered. In the subinterval 07:23:00-07:24:00 of the July 8, 2000 record, no spike was detected in the $L$ channel.

A simple fitting technique, i.e. a function of the form $f(t)=$ $C \exp \left(t / t_{\mathrm{a}}\right)$ and $f(t)=C \exp \left(-t / t_{\mathrm{d}}\right)$, was used for both the ascending and the decaying parts of the time profiles (from minimum to maximum and vice versa) in order to evaluate the parameters $t_{\mathrm{a}}$ and $t_{\mathrm{d}}$. The minimum, maximum and mean values for both ascending $\left(t_{\mathrm{a}}\right)$ and decaying $\left(t_{\mathrm{d}}\right)$ parts at 1420 and $2695 \mathrm{MHz}$ are listed in Table 3. The parameter $k=t_{\mathrm{a}} / t_{\mathrm{d}}$ expresses the ratio of the mean characteristic times, and is generally found to be near 1 with a slight asymmetry towards values smaller than 1 (see Table 3). Figures $1 \mathrm{a}$ and $1 \mathrm{~b}$ represent the scatter plots $t_{\mathrm{d}}$ versus $t_{\mathrm{a}}$ for the spikes at 1420 and $2695 \mathrm{MHz}$, respectively. The mean values in the distribution correspond to $k \approx 1$, revealing however a broad dispersion. This means that spikes with very asymmetric time profiles are quite common, i.e. spikes with fast increase and slow decrease and vice versa. Superposition of nearby spikes can hardly influence the determination of $t_{\mathrm{a}}$ and $t_{\mathrm{d}}$.

\subsubsection{Detailed study}

For this purpose 10 individual spikes (with reasonably good profile) observed on July 20, 2000 at $1420 \mathrm{MHz}$ were selected. The essential criteria were the smooth increase and decrease in the time profile. Spikes at $2695 \mathrm{MHz}$ are shorter lasting than the $1420 \mathrm{MHz}$ ones and even the $1 \mathrm{~ms}$ resolution was not considered adequate for such a detailed analysis. For the selected spikes with ideal monotonic connection among points of the spike profile we evaluated the following basic parameters: total duration $d_{0}$, half-power duration $d_{2}$ and 1/e-power duration $d_{\mathrm{e}}$ (Table 4). For the determination of the e-folding rising and decaying times $\tau$, the method proposed by 
Table 1. Selected intervals of narrowband dm-spikes and corresponding $\mathrm{H}_{\alpha}$ and GOES soft X-ray flares.

\begin{tabular}{|c|c|c|c|c|c|c|c|c|c|c|c|}
\hline \multirow[b]{2}{*}{ Event } & \multicolumn{2}{|c|}{ Radio } & \multicolumn{5}{|c|}{$\overline{\overline{\mathrm{H}_{\alpha}}}$} & \multicolumn{4}{|c|}{$\overline{\text { GOES }}$} \\
\hline & $\begin{array}{l}\text { Start } \\
{[\mathrm{UT}]}\end{array}$ & $\begin{array}{c}\text { End } \\
\text { [UT] }\end{array}$ & $\begin{array}{l}\text { Start } \\
{[\mathrm{UT}]}\end{array}$ & $\begin{array}{l}\text { Max } \\
{[\mathrm{UT}]}\end{array}$ & $\begin{array}{l}\text { End } \\
{[\mathrm{UT}]}\end{array}$ & $\begin{array}{l}\text { Optical } \\
\text { import. }\end{array}$ & Position & $\begin{array}{l}\text { Start } \\
{[\mathrm{UT}]}\end{array}$ & $\begin{array}{l}\text { Max } \\
{[\mathrm{UT}]}\end{array}$ & $\begin{array}{l}\text { End } \\
{[\mathrm{UT}]}\end{array}$ & $\begin{array}{l}\text { X-ray } \\
\text { import. }\end{array}$ \\
\hline June 06,2000 & $16: 34$ & $16: 37$ & $12: 06$ & $15: 21$ & $18: 43$ & $3 \mathrm{~B}$ & N20E18 & $14: 58$ & $15: 25$ & $15: 40$ & $\mathrm{X} 2.3$ \\
\hline July 08,2000 & $07: 23$ & $07: 59$ & 07:00 & $07: 33$ & 08:02 & SF & N17W05 & $07: 29$ & $07: 34$ & 07:39 & C6.1 \\
\hline July 12,2000 & $10: 34$ & $10: 35$ & $10: 26$ & $10: 36$ & $10: 40$ & $2 \mathrm{~B}$ & N17E25 & $10: 18$ & $10: 37$ & $10: 46$ & X1.9 \\
\hline July 20, 2000 & $13: 26$ & $13: 27$ & $13: 26$ & $13: 28$ & $13: 38$ & SF & N11E20 & $13: 16$ & $13: 23$ & $13: 33$ & C7.0 \\
\hline July 20, 2000 & $14: 56$ & $14: 58$ & $14: 54$ & $14: 57$ & $15: 18$ & SF & N11E18 & $14: 54$ & $14: 57$ & $15: 04$ & C5.1 \\
\hline March 28, 2001 & $12: 08$ & $12: 15$ & $11: 22$ & $11: 50$ & $12: 01$ & SN & N14W02 & $11: 21$ & $12: 14$ & $13: 06$ & M4.3 \\
\hline
\end{tabular}

Table 2. Detailed time intervals and basic parameters of the spikes.

\begin{tabular}{ccccccc}
\hline \hline $\begin{array}{c}\text { Event } \\
\text { No. }\end{array}$ & Data & $\begin{array}{c}\text { Time interval } \\
{[\mathrm{UT}]}\end{array}$ & $\begin{array}{c}\text { Frequency } \\
{[\mathrm{MHz}]}\end{array}$ & $\begin{array}{c}\text { Max value } \\
{[\text { SFU] }}\end{array}$ & $\begin{array}{c}\text { Min value } \\
{[\text { SFU] }}\end{array}$ & $\begin{array}{c}\text { Number of } \\
\text { spikes }\end{array}$ \\
\hline 1R & July 08, 2000 & $07: 23: 00-07: 24: 00$ & 1420 & 167.8 & 70.7 & 65 \\
2R & July 08, 2000 & $07: 32: 00-07: 34: 20$ & 1420 & 187.5 & 69.1 & 988 \\
3L & July 08, 2000 & $07: 56: 20-07: 59: 20$ & 1420 & 151.2 & 70.1 & 38 \\
3R & July 08, 2000 & $07: 56: 20-07: 59: 20$ & 1420 & 866.4 & 68.7 & 1287 \\
4L & July 12, 2000 & $10: 34: 00-10: 35: 00$ & 1420 & 396.8 & 150.8 & 708 \\
5L & July 20, 2000 & $13: 26: 00-13: 27: 00$ & 1420 & 641.1 & 96.8 & 600 \\
6L & July 20, 2000 & $14: 56: 00-14: 58: 00$ & 1420 & 1143.6 & 97.0 & 1626 \\
7L & March 28, 2001 & $12: 08: 00-12: 09: 00$ & 2695 & 163.0 & 116.0 & 117 \\
7R & March 28, 2001 & $12: 08: 00-12: 09: 00$ & 2695 & 496.7 & 123.1 & 2143 \\
8L & March 28, 2001 & $12: 10: 55-12: 11: 25$ & 2695 & 233.0 & 155.7 & 311 \\
8R & March 28, 2001 & $12: 10: 55-12: 11: 25$ & 2695 & 353.7 & 162.2 & 1007 \\
9L & March 28, 2001 & $12: 14: 10-12: 14: 50$ & 2695 & 205.8 & 161.0 & 75 \\
9R & March 28, 2001 & $12: 14: 10-12: 14: 50$ & 2695 & 381.6 & 167.4 & 1031 \\
\hline
\end{tabular}

Table 3. Detailed spike intervals and e-folding times $t_{\mathrm{a}}$ and $t_{\mathrm{d}}$ derived from statistical analysis.

\begin{tabular}{cccccccc}
\hline $\begin{array}{c}\text { Event } \\
\text { No. }\end{array}$ & $\begin{array}{c}\text { Min } t_{\mathrm{a}} \\
{[\mathrm{ms}]}\end{array}$ & $\begin{array}{c}\text { Max } t_{\mathrm{a}} \\
{[\mathrm{ms}]}\end{array}$ & $\begin{array}{c}\text { Mean } t_{\mathrm{a}} \\
{[\mathrm{ms}]}\end{array}$ & $\begin{array}{c}\text { Min } t_{\mathrm{d}} \\
{[\mathrm{ms}]}\end{array}$ & $\begin{array}{c}\text { Max } t_{\mathrm{d}} \\
{[\mathrm{ms}]}\end{array}$ & $\begin{array}{c}\text { Mean } t_{\mathrm{d}} \\
{[\mathrm{ms}]}\end{array}$ & $k=\frac{\text { mean } t_{\mathrm{a}}}{\text { mean } t_{\mathrm{d}}}$ \\
\hline 1R & 7.8 & 77.7 & 32.3 & 13.5 & 121.3 & 40.7 & 0.79 \\
2R & 19.1 & 167.9 & 55.4 & 21.9 & 232.5 & 59.1 & 0.94 \\
3L & 21.5 & 119.4 & 49.9 & 31.2 & 121.8 & 54.6 & 0.91 \\
3R & 4.4 & 118.1 & 30.3 & 6.7 & 145.7 & 32.3 & 0.94 \\
4L & 24.9 & 243.7 & 82.7 & 29.1 & 228.5 & 82.2 & 1.01 \\
5L & 7.1 & 233.2 & 41.6 & 8.3 & 122.6 & 42.5 & 0.98 \\
6L & 5.8 & 205.2 & 47.1 & 7.3 & 218.8 & 49.3 & 0.96 \\
7L & 11.9 & 129.0 & 48.1 & 19.0 & 140.9 & 51.2 & 0.94 \\
7R & 3.7 & 124.5 & 28.5 & 4.6 & 172.4 & 30.9 & 0.92 \\
8L & 18.9 & 169.5 & 59.1 & 16.8 & 165.6 & 64.8 & 0.91 \\
8R & 8.7 & 134.4 & 41.1 & 11.4 & 193.1 & 45.9 & 0.90 \\
9L & 44.5 & 226.6 & 93.9 & 50.0 & 235.9 & 98.6 & 0.95 \\
9R & 15.6 & 209.8 & 59.6 & 10.3 & 217.3 & 62.8 & 0.95 \\
\hline
\end{tabular}

Güdel \& Benz (1990) has been used. However, we realized that the results are very sensitive to the estimation of the background level $f_{0}$. Therefore, a more precise technique which fits the observed data with three free parameters was used. We assumed an exponential function of the form

$f(t)=\exp \left[\left(t+t_{0}\right) / \tau\right]+f_{0}$,

where $t_{0}, \tau$, and $f_{0}$ are the fitting parameters. This formula yields the growth and the decay times $(\tau$ and $-\tau)$ together with the "background" level for the ascending and the decaying part separately (Table 4). The two backgrounds $f_{0}$ for the same spike do not necessarily coincide. Then, after subtracting the "background" flux density, the logarithmic derivative can be computed and it results inversely proportional to the growth and the decay time, i.e. $\tau=\left(\frac{\mathrm{d}}{\mathrm{d} t} \log \left(f(t)-f_{0}\right)\right)^{-1}$, respectively.

Generally (in 9 out of 10 cases) a plateau which indicates the exponential trend was recognized in the decaying part of spikes (see the examples in Fig. 2 and in Table 4). However, 
Table 4. Parameters for the July 20, 2000 selected spikes at $1420 \mathrm{MHz}$ ( $d_{0}=$ total duration, $d_{2}=$ half-power duration, $d_{\mathrm{e}}=1 / \mathrm{e}$-power duration, $\tau=$ growth time, $-\tau=$ decay time, $f_{0}=$ background, $T=$ derived temperature).

\begin{tabular}{ccccccccc}
\hline \hline & & \multicolumn{4}{c}{ Ascending part } & \multicolumn{3}{c}{ Decaying part } \\
$\begin{array}{c}\text { Spike interval } \\
{[\mathrm{UT}]}\end{array}$ & $\begin{array}{c}d_{0} \\
{[\mathrm{~ms}]}\end{array}$ & $\begin{array}{c}d_{2} \\
{[\mathrm{~ms}]}\end{array}$ & $\begin{array}{c}d_{\mathrm{e}} \\
{[\mathrm{ms}]}\end{array}$ & $\begin{array}{c}\tau \\
{[\mathrm{ms}]}\end{array}$ & $\begin{array}{c}f_{0} \\
{[\mathrm{SFU}]}\end{array}$ & $\begin{array}{c}-\tau \\
{[\mathrm{ms}]}\end{array}$ & $\begin{array}{c}f_{0} \\
{[\mathrm{SFU}]}\end{array}$ & {$[\mathrm{K}]$} \\
\hline 13:26:32.925-13:26:32.950 & 18 & 8 & 10 & - & & 6.8 & 110.1 & $4.6 \times 10^{6}$ \\
13:26:46.930-13:26:46.950 & 16 & 8 & 9 & 4.5 & 85.8 & 8.4 & 97.7 & $5.3 \times 10^{6}$ \\
13:26:46.951-13:26:46.970 & 14 & 6 & 8 & - & & 2.4 & 135.3 & $2.3 \times 10^{6}$ \\
14:57:14.875-14:57:14.898 & 13 & 6 & 8 & - & & 5.2 & 245.7 & $3.9 \times 10^{6}$ \\
14:57:16.660-14:57:16.683 & 14 & 5 & 7 & - & & 3.7 & 291.2 & $3.1 \times 10^{6}$ \\
14:57:20.178-14:57:20.210 & 21 & 7 & 9 & 3.6 & 203.1 & 5.8 & 209.8 & $4.2 \times 10^{6}$ \\
14:57:20.897-14:57:20.922 & 19 & 7 & 9 & 7.5 & 134.1 & 3.1 & 325.4 & $2.7 \times 10^{6}$ \\
14:57:22.560-14:57:22.592 & 25 & 11 & 13 & 3.2 & 269.7 & - & & - \\
14:57:24.150-14:57:24.190 & 25 & 10 & 12 & - & & 4.7 & 184.5 & $3.6 \times 10^{6}$ \\
14:57:26.195-14:57:26.215 & 16 & 7 & 9 & - & & 5.3 & 200.6 & $3.9 \times 10^{6}$ \\
\hline
\end{tabular}
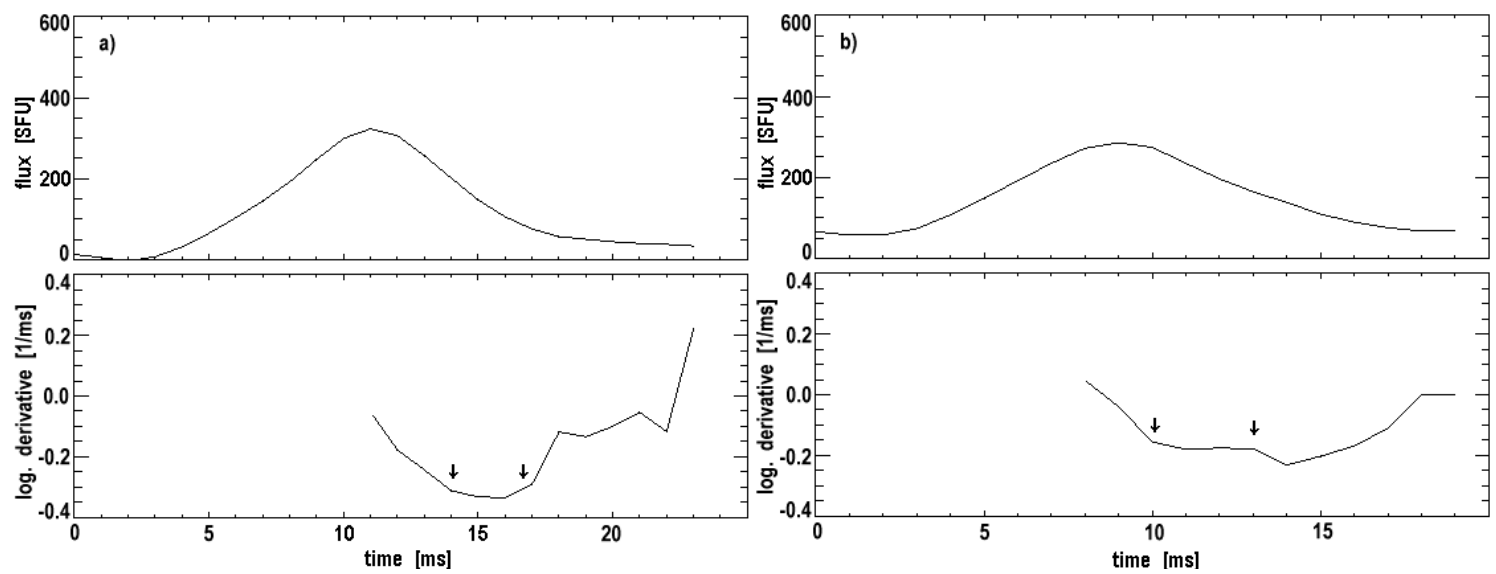

Fig. 2. Example of time profile of two spikes at $1420 \mathrm{MHz}$ and their logarithmic derivative during their decaying part: spike recorded at: a) 14:57:20.897-14:57:20.922 and b) 14:57:26.195-14:57:26.215 UT. The arrows indicate the derived plateau interval.
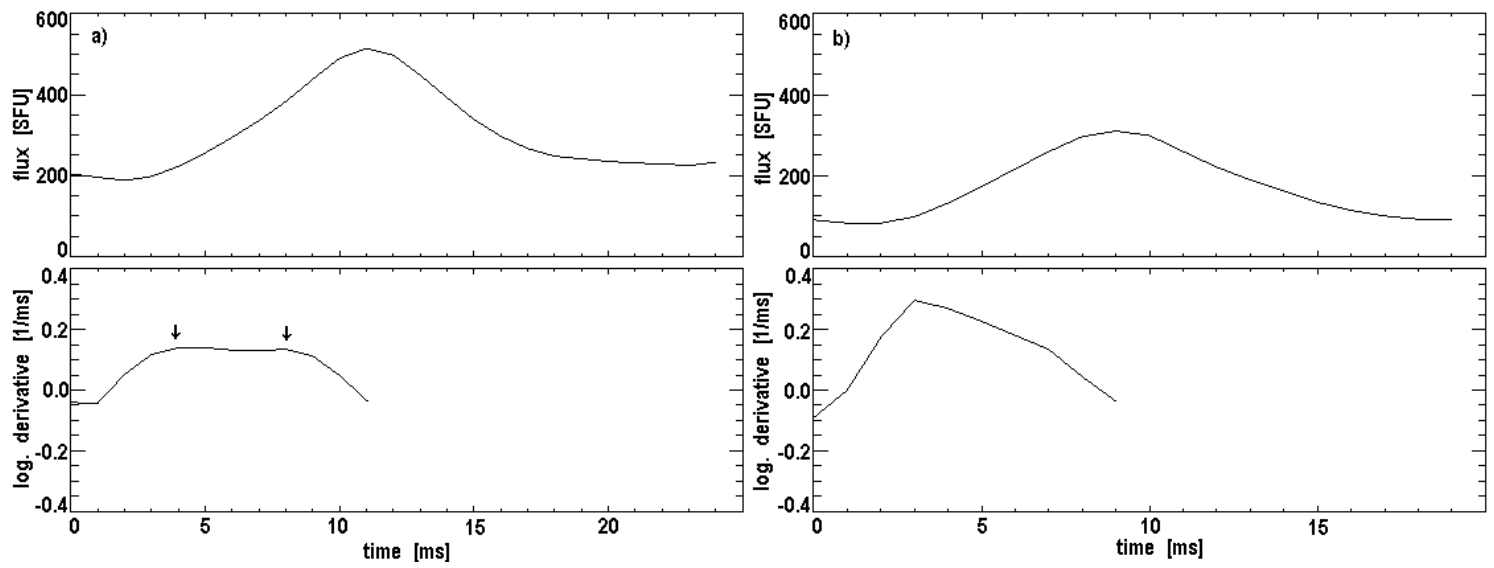

Fig. 3. Example of time profile of two spikes at $1420 \mathrm{MHz}$ and their logarithmic derivative during their ascending part: same spikes as in Fig. 2. The arrows indicate the derived plateau interval.

spikes exist (4 out of 10) where a similar plateau was found also during the ascending part (see Fig. 3a). In this case, both the instability which generates the spike, and the plasma wave absorption are described by exponential functions. In any way, during the whole spike the collisional absorption is present and it depends on the plasma temperature $\left(\sim T^{-3 / 2}\right)$. We used the exponential trend during the decaying part for the coronal temperature estimations (last column in Table 4). Figure 3b shows a case without plateau in the ascending part of the spike. 
Table 5. Half-power duration and polarization at maximum for selected spikes at $1420 \mathrm{MHz}$ on July 20, 2000 and at $2695 \mathrm{MHz}$ on June 6, 2000 and March 28, 2001.

\begin{tabular}{ccccc}
\hline \hline Event & $\begin{array}{c}\text { Spike interval } \\
{[\mathrm{UT}]}\end{array}$ & $\begin{array}{c}\text { Number of spikes } \\
\text { July 20, 2000 }\end{array}$ & $\begin{array}{c}\text { Half-power duration } \\
{[\mathrm{ms}]}\end{array}$ & $\begin{array}{c}\text { Polarization } \\
{[\%]}\end{array}$ \\
\hline July 20, 2000 & $13: 26: 19-13: 26: 21$ & 36 & $9.4 \pm 1.8$ & $0.4 \mathrm{~L} \pm 5.1$ \\
July 20, 2000 & $13: 26: 37-13: 26: 34$ & 13 & $9.6 \pm 2.0$ & $0.8 \mathrm{~L} \pm 3.6$ \\
July 20, 2000 & $13: 26: 40-13: 26: 43$ & 19 & $10.4 \pm 2.7$ & $1.0 \mathrm{R} \pm 4.0$ \\
July 20, 2000 & $13: 26: 44-13: 26: 48$ & 49 & $9.4 \pm 2.0$ & $1.2 \mathrm{R} \pm 5.6$ \\
July 20, 2000 & $14: 56: 36-14: 56: 45$ & 15 & $8.8 \pm 2.1$ & $1.4 \mathrm{R} \pm 4.6$ \\
July 20, 2000 & $14: 56: 51-14: 57: 02$ & 21 & $10.4 \pm 2.4$ & $2.9 \mathrm{~L} \pm 4.3$ \\
July 20, 2000 & $14: 57: 02-14: 57: 16$ & 108 & $9.0 \pm 1.9$ & $3.5 \mathrm{~L} \pm 5.4$ \\
July 20, 2000 & $14: 57: 16-14: 57: 31$ & 191 & $8.8 \pm 2.1$ & $1.6 \mathrm{~L} \pm 5.6$ \\
July 20, 2000 & $14: 57: 31-14: 57: 38$ & 14 & $8.9 \pm 2.1$ & $1.1 \mathrm{~L} \pm 3.9$ \\
\hline Mean values & - & 506 & $7.6 \pm 1.8$ & $4.1 \mathrm{R} \pm 4.5$ \\
\hline June 6, 2000 & $16: 34: 48-16: 35: 27$ & 81 & 9.0 & $0.7 \mathrm{~L}$ \\
June 6, 2000 & $16: 36: 01-16: 37: 06$ & 111 & $5.6 \pm 0.9$ & $70.9 \mathrm{R} \pm 7.5$ \\
June 6, 2000 & $16: 37: 21-16: 37: 30$ & 30 & $5.1 \pm 0.9$ & $72.7 \mathrm{R} \pm 6.0$ \\
March 28, 2001 & $12: 08: 04-12: 08: 10$ & 114 & $5.4 \pm 0.9$ & $70.3 \mathrm{R} \pm 9.7$ \\
March 28, 2001 & $12: 08: 10-12: 08: 20$ & 151 & $4.3 \pm 0.7$ & $71.8 \mathrm{R} \pm 4.4$ \\
March 28, 2001 & $12: 08: 25-12: 08: 48$ & 75 & $4.5 \pm 0.7$ & $75.1 \mathrm{R} \pm 3.6$ \\
March 28, 2001 & $12: 10: 58-12: 11: 18$ & 107 & $4.8 \pm 0.6$ & $73.0 \mathrm{R} \pm 5.9$ \\
March 28, 2001 & $12: 14: 15-12: 14: 40$ & 130 & $5.4 \pm 0.6$ & $39.8 \mathrm{R} \pm 14.9$ \\
\hline Mean values & - & 799 & $5.7 \pm 0.7$ & $70.5 \mathrm{R} \pm 11.1$ \\
\hline
\end{tabular}
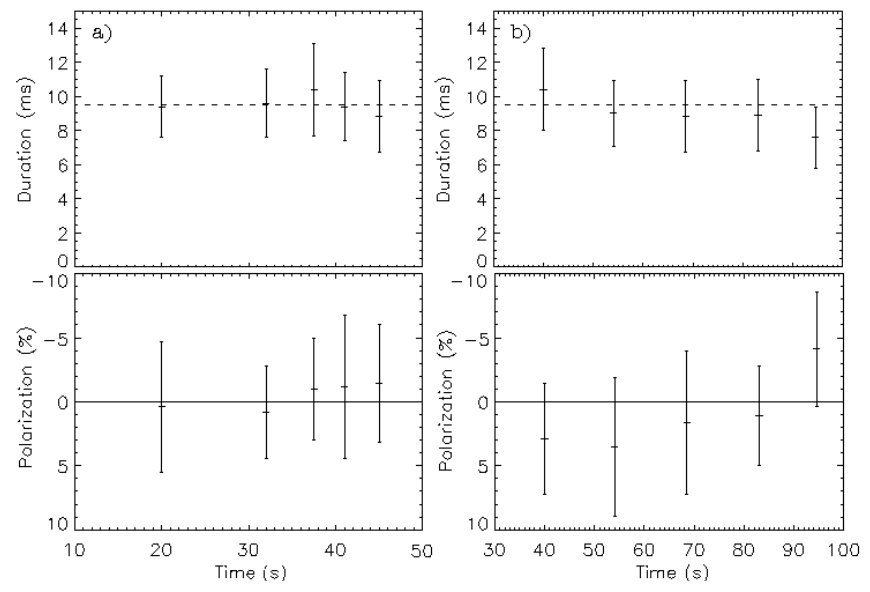

Fig. 4. Half-power duration and polarization of spikes recorded at $1420 \mathrm{MHz}$ during July 20, 2000. Positive sign means L-handed polarization. For a) the starting time was 13:26:00 and for b) 14:56:00 UT. The dashed line shows the duration given by the formula reported by Güdel \& Benz (1990).

\subsection{Spike duration and polarization}

We evaluated the half-power duration of some hundreds of spikes as well as their polarization degree and the possible delay of one polarimetric component with respect to the other.

\subsubsection{Spikes at $1420 \mathrm{MHz}$}

We selected 10 groups of spikes on July 20, 2000 (see Table 5). These groups were divided into smaller subsections (generally lasting $1 \mathrm{~s}$ ) in order to detect changes of the evaluated parameters. Only spikes with intensity stronger than about $60 \mathrm{SFU}$ (above the background) were selected, few of them exceeded 500 SFU. Spikes showing complex shape or superposition were not considered. For the selected spikes we derived their duration at half-power and the polarization value at the maximum. Mean values of half-power durations and of polarization values are summarized in Table 5. Another interesting parameter comes from the evaluation either of the clock-wise $(\mathrm{CW})$ or of the anti-clock-wise (ACW) sense evolution in time of a single event when we plot its contemporaneous $\mathrm{R}$ versus $\mathrm{L}$ values (Zlobec \& Karlický 1998). This sense is related to the delay of one polarimetric component with respect to the other; in particular an ACW (CW) spike has its R (L) component delayed. The characteristics of the spikes were practically the same during all the 10 groups.

The distribution of the duration at half power for the different groups and subsections, when the number of spikes was representative, always peaked at 8,9 or $10 \mathrm{~ms}$. The overall mean value was $9.0 \mathrm{~ms}$. It is important to note that the duration we obtained fits very well with the value obtained using the formula given by Güdel \& Benz (1990), i.e. $9.5 \pm 1.0 \mathrm{~ms}$, which was derived for spikes up to about $1 \mathrm{GHz}$ (upper parts of Fig. 4).

The polarization of the considered spikes was close to zero and remained nearly constant during the considered interval. The global mean value was $0.7 \% \mathrm{~L}$. Mean values in different groups were limited between $4.1 \%$ in the R-sense and $3.5 \%$ in the L-sense (lower parts of Fig. 4).

\subsubsection{Spikes at $2695 \mathrm{MHz}$}

At $2695 \mathrm{MHz}$ the spikes were weaker and the groups in which they appeared lasted shorter than those at $1420 \mathrm{MHz}$. Therefore, we selected groups of spikes during two 

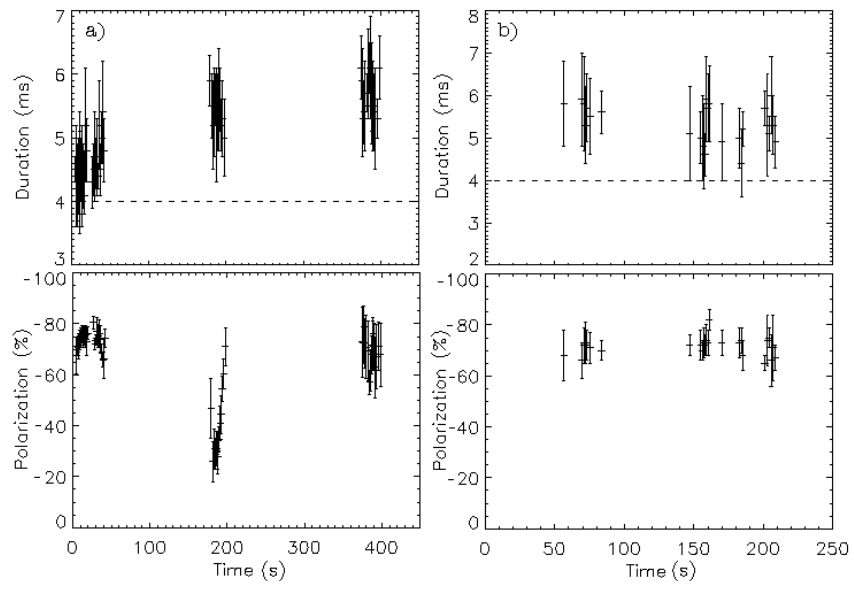

Fig. 5. Half-power duration and polarization (R-handed) of spikes recorded at $2695 \mathrm{MHz}$. a) March 28, 2001 data with starting time at 12:08:00 and b) June 6, 2000 data with starting time at 16:34:00 UT. The dashed line shows the duration value analogously as in Fig. 4.

days: June 6, 2000 and March 28, 2001 (see Table 5). Similarly as at $1420 \mathrm{MHz}$ these groups were divided into subsections generally lasting $1 \mathrm{~s}$. Only spikes that were not superposed by other bursts and with intensity stronger than about 50 SFU were selected.

The most interesting groups with numerous spikes were recorded on March 28, 2001 at 12:08:04-12:08:48. During $44 \mathrm{~s}$ we selected 340 bursts that were divided into 26 subsections. During the first two groups recorded on that day the most probable duration (time interval 12:08:04-20) was in the range 3.7$4.6 \mathrm{~ms}$, meanwhile afterwards (12:08:25-48) the values were between 4.5 and $4.8 \mathrm{~ms}$, which is a remarkable difference. Except for the first three groups, the mean values were always longer than $5.0 \mathrm{~ms}$ (upper parts of Fig. 5). The earliest spikes of March 28, 2001 match the result given by the Güdel \& Benz (1990) formula $(4.0 \pm 0.7 \mathrm{~ms})$ which was established considering spikes up to about $1 \mathrm{GHz}$; the other spikes showed a comparatively longer duration. The spikes recorded on June 6, 2000 with their mean duration longer than $5 \mathrm{~ms}$ confirm the existence of relatively long-lasting spikes in respect to the result given by the mentioned formula.

Recently Wang et al. (2002) reported spikes in the range $2.6-3.8 \mathrm{GHz}$, and their lifetime was rather long (i.e. 23.8-29.4 ms).

As the duration, at half power, was in the range $4-5 \mathrm{~ms}$, the whole spike profile was sampled by at least 10 measurements, which ensures that the digitization rate was appropriate for the evaluation of the parameters we considered (see Isliker \& Benz 2001). In order to verify that there is no relationship between duration and intensity, we divided the duration interval in three sets and computed for each of them the mean value of their intensities: we practically realized the same mean value in all three cases. This outcome indicates that intensity and duration are independent parameters.

During the majority of the considered subsections the polarization was about $70 \% \mathrm{R}$. In the group recorded on March 28, 2001 during 12:10:58-12:11:18 the polarization was initially about $45 \% \mathrm{R}$, then it dropped to $26 \% \mathrm{R}$ and finally increased up to $70 \% \mathrm{R}$ (lower parts of Fig. 5). We did not find any correlation between duration and polarization. This is in accordance with the results reported in Zlobec \& Karlický (1998) at frequencies lower than $1 \mathrm{GHz}$.

\subsection{Delay between the polarimetric components}

In order to find out the possible delay between the polarimetric components we plotted the contemporaneous $\mathrm{R}$ versus $\mathrm{L}$ values. In this representation, a delay between the polarimetric components leads to the formation of a loop. In particular a wider loop means a larger delay (see Zlobec \& Karlický 1998).

At $1420 \mathrm{MHz}$ during July 20, 2000, when the polarization was practically zero, we did not find sequences (more than 5 events) of spikes showing a systematic trend in the delay between the two polarimetric components. The delay, when present, was just of a fraction of a millisecond, generally in the range $0.1-0.3 \mathrm{~ms}$. The indetermined cases were the most numerous (39\%), followed by ACW cases, i.e. the R component was delayed $(38 \%)$ and the $\mathrm{CW}$ cases were the most rare $(23 \%)$. Due to the fact that sequences of the same type of delay were not present, we concluded that there was a statistical predominance of R-delayed spikes, that was mainly seen in the interval 13:26:37-13:26:48 and after 14:57:16 up to the end.

At $2695 \mathrm{MHz}$ during March 28, 2001, in the time interval 12:08:05-12:08:48 the polarization was rather strong in R-sense. A predominance of indetermined cases was present (43\%), followed by ACW cases (33\%) and CW cases $(25 \%)$. The higher number of ACW events in respect to $\mathrm{CW}$ cases showed a more common presence of R-delayed component, i.e. the strongest component was delayed and that is not in accordance with the result by Benz \& Pianezzi (1997). During the following interval (12:10:58-12:11:18) the R-polarization was weaker and the ACW cases were still more numerous (50\%), meanwhile the indetermined and $\mathrm{CW}$ cases were statistically almost equal. In particular during one second (12:11:08) nine ACW cases formed a sequence, but four seconds after seven CW events were following one another. In the third interval (12:14:15-12:14:40) the polarization was quite strong in the R-sense, but the statistical result regarding the delay was in the other sense: $39 \%$ were the $\mathrm{CW}$ and the indetermined cases, and 22\% the ACW cases. During June 6, 2000, when the spikes were also strongly R-polarized, the situation was: the CW cases were $37 \%$, the ACW cases $32 \%$ and the indetermined ones $31 \%$.

There was also no connection between the variation of the polarization degree (the same was also valid for the duration) and the presence of cases in which the delay of one polarimetric component in respect to the other was found.

\section{Nonlinear analysis}

\subsection{Methods}

For the purpose of nonlinear analysis spikes intervals with a high occurrence rate and high intensities were selected. For the July 20, 2000 and March 28, 2001 intervals, 10 and 6 groups, respectively, were selected (see Tables 6 and 7). 
Table 6. Summary of the nonlinear analysis for the selected parts of the July 20, 2000 event (R-component of the spikes at $1420 \mathrm{MHz}$ ). We list the outcome of the correlation dimension analysis ("negative" indicates that no low-dimensional determinism is detected), the "surrogate subsequence" is a part of the original sequence minimizing the end-to-end mismatch; and the outcome from the nonlinearity test ("positive" indicates evidence for nonlinearity on a 95\% significance level). "PE" denotes the nonlinear predictability measure, "REV" indicates that the time-reversibility measure gave a positive result for the surrogate data test.

\begin{tabular}{ccccl}
\hline \hline $\begin{array}{c}\text { Spike interval } \\
{[\mathrm{UT}]}\end{array}$ & Low dimension & \multicolumn{2}{c}{ Surrogate subsequence } & Nonlinearity \\
{$[\%]$} & & [points] & \\
\hline 13:26:18.0-13:26:22.0 & negative & 100.0 & 4000 & positive (PE) \\
13:26:30.0-13:26:34.5 & negative & 97.2 & 4375 & negative \\
13:26:34.5-13:26:39.0 & negative & 97.2 & 4375 & negative \\
13:26:39.0-13:26:43.5 & negative & 97.2 & 4375 & positive (PE) \\
13:26:43.5-13:26:48.0 & negative & 96.0 & 4321 & positive (REV) \\
14:56:34.0-14:56:48.0 & negative & 98.7 & 13825 & positive (PE) \\
14:56:48.0-14:57:02.0 & negative & 74.1 & 10369 & positive (REV) \\
14:57:02.0-14:57:16.0 & negative & 96.4 & 13501 & positive (PE) \\
14:57:16.0-14:57:30.0 & negative & 96.4 & 13501 & positive (PE) \\
14:57:30.0-14:57:44.0 & negative & 93.7 & 13124 & positive (PE) \\
\hline
\end{tabular}

Table 7. Summary of the nonlinear analysis for the selected parts of the March 28, 2001 event (R-component of the spikes at $2695 \mathrm{MHz}$ ). For a detailed description see Table 6.

\begin{tabular}{ccccl}
\hline \hline $\begin{array}{c}\text { Spike interval } \\
{[\mathrm{UT}]}\end{array}$ & Low dimension & \multicolumn{2}{c}{ Surrogate subsequence } & Nonlinearity \\
{$[$ [points] } & \\
\hline 12:08:07.5-12:08:19.5 & negative & 100.0 & 12000 & positive (PE, REV) \\
12:08:31.0-12:08:44.0 & negative & 99.7 & 12961 & positive (PE, REV) \\
12:10:59.0-12:11:03.0 & negative & 97.2 & 3889 & positive (PE, REV) \\
12:11:05.0-12:11:18.0 & negative & 99.7 & 12961 & positive (REV) \\
12:14:16.0-12:14:22.0 & negative & 97.2 & 5833 & negative \\
12:14:28.0-12:14:32.0 & negative & 97.2 & 3888 & negative \\
\hline
\end{tabular}

The correlation dimension is one out of various definitions of fractal dimensions. It was introduced by Grassberger \& Procaccia $(1983 a, b)$ to determine fractal dimensions from time series. The most important method to reconstruct the phase space of the dynamical system from the measured time series is the technique of time delayed coordinates (Takens 1981). From the one-dimensional time series $\left\{x\left(t_{i}\right)\right\}$ an $m$-dimensional phase space $\left\{\xi_{i}\right\}$ is constructed using the prescription

$\xi_{i}=\left\{x\left(t_{i}\right), x\left(t_{i}+\tau\right), \ldots, x\left(t_{i}+(m-1) \tau\right)\right\}$,

where $\tau$ is the time delay. Most commonly, $\tau$ is chosen as the auto-correlation time or as the first minimum of the mutual information (Fraser \& Swinney 1986). The correlation dimension is most efficiently computed by the correlation sum $C(r, m)$ which is given by the normalized number of pairs of points within a distance $r$ in the $m$-dimensional embedding space,

$C(r, m)=\frac{1}{N_{\text {pairs }}} \sum_{i=1}^{\mathrm{N}-W} \sum_{j=i+W}^{\mathrm{N}} \Theta\left(r-\left\|\xi_{i}-\xi_{j}\right\|\right)$.

$N$ denotes the length of the time series, $N_{\text {pairs }}$ gives the actual number of pairs of points covered by the double sum, and $\Theta$ is the Heaviside step function. The correction term $W$ is necessary in order to exclude temporally correlated points from the sum; the length of the window $W$ should not be smaller than the auto-correlation time in order to avoid spurious dimension estimates (Theiler 1986). For small distances $r$, the correlation sum $C(r, m)$ is expected to scale with a power of $r$. If the embedding dimension $m$ exceeds the box-dimension of the attractor, the scaling exponent gives the correlation dimension $D_{\mathrm{c}}$,

$C(r, m) \propto r^{D_{\mathrm{c}}}, \quad D_{\mathrm{c}}=\lim _{r \rightarrow 0} \frac{\ln C(r, m)}{\ln r}$.

Thus, if $D_{\mathrm{c}}$, calculated for increasing embedding dimension $m$, reaches a saturation value for a relatively small $m$, it provides evidence for low-dimensional determinism underlying the analyzed time series. A maximum likelihood estimator of the correlation dimension is given by the Takens-Theiler estimator (Takens 1985; Theiler 1988):

$D_{\mathrm{TT}}(r)=\frac{C(r)}{\int_{0}^{r} \frac{C\left(r^{\prime}\right)}{r^{\prime}} \mathrm{d} r^{\prime}}$.

For details on the computation see the paper by Hegger et al. (1999), in which a description of the used TISEAN (Nonlinear Time Series Analysis) package is given. The relevant caveats concerning the calculation and interpretation of correlation dimensions is reviewed by Grassberger et al. (1991).

Correlation dimensions are, in principle, an appropriate tool to test for nonlinear deterministic signatures in measurement time series. However, if, e.g., the determinism is not low-dimensional, or if additional noise is present (even small amounts, see Schreiber \& Kantz 1995), or if the data cannot be expected to be in a stationary state during the measurement period, then a possible determinism cannot be detected by this method. All three possibilities presumably apply to solar radio 
flux time series, which represent real world data. As argued, e.g., by Hegger et al. (1999), the bulk of real world time series belongs to neither of the extreme scenarios, nonlinear fully deterministic versus classical linear stochastic processes. Most often, they reflect nonlinear responses and effectively stochastic processes at the same time. Thus, it is advisable to test if nonlinearity is present in the data at all.

The degree of nonlinearity can be measured in different ways. However, since all measures of nonlinearity show fluctuations but the distributions are not analytically available, it is necessary to apply Monte Carlo techniques in order to evaluate the significance of the results (e.g., Hegger et al. 1999). A very important method in this context is represented by surrogate data (Theiler et al. 1992). In a surrogate data test the value of a nonlinear measure derived from the data is compared to a number of randomized samples, the surrogates. Non-Gaussianity is the simplest kind of nonlinear signature. However, it may have a trivial reason, insofar the data might have been distorted in the measurement process (Theiler et al. 1992; Hegger et al. 1999). To test against this null hypothesis of a linear stochastic process or of a nonlinear transformation of such a process, we apply the amplitude adjusted Fourier transform method proposed by Theiler et al. (1992) and improved by Schreiber \& Schmitz (1996). In this case, the surrogates are constrained to have the same distribution of values and the same Fourier amplitudes as the original data (the method of surrogate time series was reviewed by Schreiber \& Schmitz 2000).

For the purpose of nonlinearity testing, any nonlinear measure can be used. However, the method is most successful if test statistics that are powerful in the discrimination of linear dynamics and weakly nonlinear signatures are used. In the present study we utilize two different nonlinearity measures suggested by the study of Schreiber \& Schmitz (1999), nonlinear predictability and time-reversibility. The nonlinear predictability test statistics is given by the nonlinear prediction error with respect to a locally constant predictor $F$, defined as (Schreiber \& Schmitz 1999):

$t^{\mathrm{PE}}(m, \tau, r)=\left(\sum\left[\xi_{i+1}-F\left(\xi_{i}\right)\right]^{2}\right)^{1 / 2}$.

The prediction over one time step is performed by averaging over the future values of all neighboring delay vectors $\xi_{i}$, which are closer than a distance $r$ in dimension $m$.

A time series is said to be reversible if its probabilistic properties are invariant with respect to time reversal. Since linear stochastic processes are fully characterized by their power spectrum which does not contain any information on the direction of time, asymmetry under time reversal is an indicator of nonlinearity (Diks et al. 1995). A simple test statistics to estimate deviations from time-reversibility is given by (Schreiber \& Schmitz 1999, 2000):

$t^{\mathrm{REV}}(\tau)=\frac{1}{N-\tau} \sum_{i=\tau+1}^{N}\left(x_{i}-x_{i-\tau}\right)^{3}$.

As periodicity artifacts can lead to spurious results in the application of Fourier based surrogates, it is important to select an appropriate subsequence of the time series before creating surrogates (Schreiber \& Schmitz 2000). In particular, any mismatch in value and phase between the beginning and the end of the time series poses problems. The relevant formulae used to minimize the so-called end-to-end mismatch are given in the appendix of the paper by Schreiber \& Schmitz (2000).

If the results of the nonlinear measures of an ensemble of surrogate data are significantly different from those derived from the original data, the null hypothesis that the obtained results are caused by a linear stochastic process can be rejected. To quantify the significance of the test, we follow the rank-order test described in Schreiber \& Schmitz (2000). First, a residual probability $\alpha$ of a false rejection of the null hypothesis is selected, corresponding to a level of significance of $(1-\alpha)$. Then, for a one-sided test $M=1 / \alpha-1$ (for a two-sided test $M=2 / \alpha-1)$ surrogate data sets are created. Thus, for a significance requirement of $95 \%$, at least 19 and 39 surrogate times series are needed for one- and two-sided hypothesis tests, respectively.

\subsection{Results}

In the present study, we calculated the correlation sum and the correlation dimension up to the embedding dimension $m=10$. For the determination of the time delay $\tau$ we applied the first minimum of the mutual information as well as the first minimum of the auto-correlation function. In order to exclude any bias due to temporal correlations, we have chosen the Theilerwindow $W$ as at least two times the auto-correlation time. For the determination of the correlation dimension from the correlation sum, the Takens-Theiler maximum likelihood estimator was used. We analyzed the overall time series as well as the selected subsections.

Furthermore, for each of the time series under study we determined suitable subsequences for the surrogate data set by minimizing the weighted average of the end-to-end mismatch in value and phase. For this purpose, the length of the subsequence under study is successively decreased, only considering lengths which can be factorized with factors 2, 3 and 5, and for each length the optimal time offset is determined (see Schreiber \& Schmitz 2000). By applying this procedure we derived those subsections (3rd column in Tables 6 and 7) of the time series which yielded the most improved value for the end-to-end mismatch. From this subsequences, the nonlinear prediction error (Eq. (8)) and the time-reversibility statistics (Eq. (9)) were derived. For both measures a surrogate data test was applied on a $95 \%$ significance level. Concerning the nonlinear prediction errors, it is expected that nonlinearity in the data results in lower values. Thus, a one-sided hypothesis test was applied, resulting in a sample of 19 surrogates. In the case of the time-reversibility measure, nonlinearity leads to a test statistic that is either smaller or larger than those of the surrogates. For this test statistics, we applied a two-sided test, i.e. 39 surrogates were created. In principle, the actual choice of the free parameters in the determination of the nonlinear measures (cf. Eqs. (8) and (9)) does not affect the surrogate data test, as long as the same parameters are used for the original time series and for the surrogates. We applied three different 


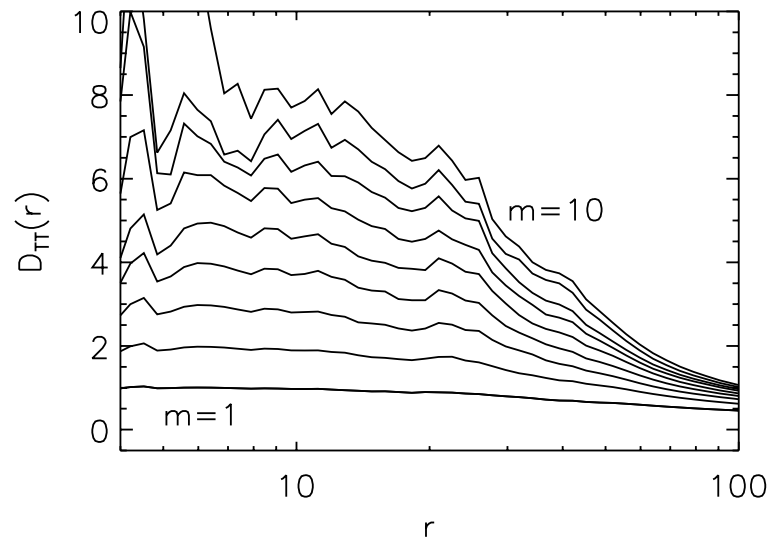

Fig. 6. Correlation dimension for embedding dimension $m=1$ to $m=10$ applying the Takens-Theiler maximum likelihood estimator, $D_{\text {TT }}$, derived from the March 28, 2001 event (12:10:59.0-12:11:03.0). The increase of $D_{\text {TT }}$ with increasing embedding dimension $m$ (in the plateau region) is in contrast to low-dimensional determinism.

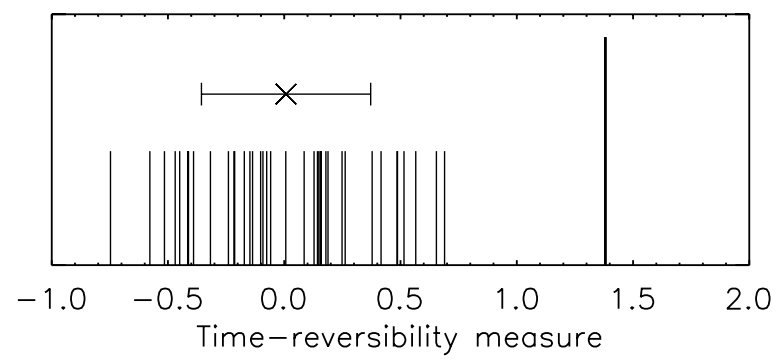

Fig. 7. Outcome of the surrogate data test for the March 28, 2001 event (12:10:59.0-12:11:03.0), using the time-reversibility measure. The value derived from the original data is plotted with an impulse longer than the values derived from the surrogate time series. The mean and the standard deviation for the surrogate sample are also plotted (represented by an asterisk with error bar). Since the value derived from the original time series lies outside from those derived from the 39 surrogates, we have evidence for nonlinearity in the data on a $95 \%$ significance level.

sets of free parameters for each nonlinear measure. The results for the July 20, 2000 and the March 28, 2001 intervals are summarized in Tables 6 and 7, respectively. In Fig. 6 an example for the correlation dimension analysis using the TakensTheiler estimator is shown. For none of the analyzed time series indications for the presence of low-dimensional determinism is found. This finding suggests that the dynamics of narrowband dm-spikes cannot be described by a low-dimensional system of, say, $D_{\mathrm{C}} \lesssim 5$, which is in agreement with previous studies (Isliker 1992; Isliker \& Benz 1994; Mészárosová et al. 2000; Veronig et al. 2000). Since we have used exceptionally high time resolution measurements, which sufficiently cover the temporal profile of individual spikes, it is suggested that this outcome is related to the intrinsic spikes dynamics rather than to limitations of the measurement process.

On the other hand, for 12 out of 16 investigated sequences the surrogate data analysis provides evidence that the time series do not represent a Gaussian linear stochastic process or a nonlinear transformation of a Gaussian process by a static measurement function. This finding indicates that (at least

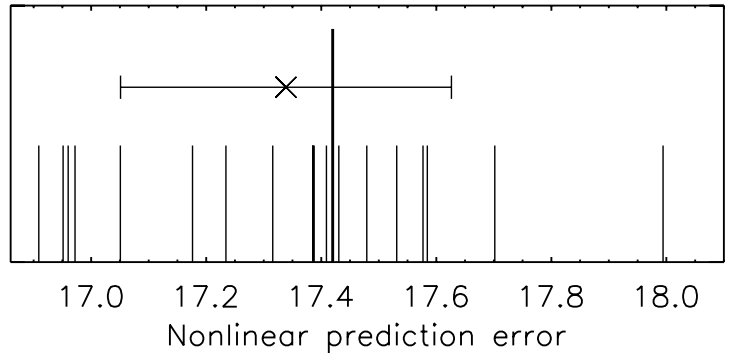

Fig. 8. Outcome of the surrogate data test for the July 20, 2000 event (13:26:30.0-13:26:34.5), using the nonlinear prediction error. Since the value derived from the original data is not smaller than those derived from the 19 surrogates, the null hypothesis of a linear stochastic process cannot be rejected.

weak) nonlinearity is present in these time series. In Figs. 7 and 8 two sample outcomes from the surrogate data test are illustrated.

A similar result has been obtained for 4 selected spikes time series by Veronig et al. (2000), however using a set-up of the surrogate data test less refined than in the present study. Schwarz et al. (1993) applied methods of symbolic dynamics to dynamic spectra of narrowband spikes, interpreting the spikes appearance in the frequency-time domain as spatio-temporal patterns. These authors found that the simultaneous appearance of spikes at different frequencies is not a purely stochastic phenomenon but might be caused by a nonlinear deterministic system or by a Markov process. This result was interpreted as being compatible with a scenario in which spikes at nearby locations are simultaneously triggered by a common exciter.

\section{Discussion and conclusions}

\section{a) Association with flares}

Four of the six associated optical flares were subflares and spikes were generally present during their rising phase (Table 1). In two cases the associated flares were of high importance. During the lifetime of the most powerful flare (type 3B) spikes (at $2695 \mathrm{MHz}$ ) appeared during its decay phase. During July 8, 2000 several groups of spikes were present, and the strongest was just the last one, i.e. in the late decay phase. In all cases the associated flares were located near the disk center and the spike characteristics were found to be independent of the importance as well as of the phase of the associated $\mathrm{H}_{\alpha}$ flare and X-ray burst.

\section{b) Time profiles}

According to the statistical study the ratio of the values of the characteristic e-folding times during the ascending and the decaying part of individual spikes correspond to $k_{\text {mean }}=t_{\mathrm{a}} / t_{\mathrm{d}} \approx 1$, however the $k$ values show a broad dispersion. As $t_{\mathrm{d}} \sim T^{-3 / 2}$ the question arises: why the mean values $t_{\mathrm{a}}$ and $t_{\mathrm{d}}$ are approximately equal. There are theories about the emission of narrowband dm-spikes (e.g. Bárta \& Karlický 2001) in which also their growth rate is proportional to $T^{-3 / 2}$. However, the collisional damping and the instability growth rate are generally not the same. Moreover, the collisional damping is always present. 
Therefore, the ascending part of spikes is the result of the instability and also of the damping $\left(f \sim \exp \left(t / t_{\mathrm{a} *}\right) \exp \left(-t / t_{\mathrm{d}}\right) \sim\right.$ $\exp \left(t / t_{\mathrm{a}}\right)$, i.e. $1 / t_{\mathrm{a}} \sim 1 / t_{\mathrm{a} *}-1 / t_{\mathrm{d}}$, where $t_{\mathrm{a} *}$ is the instability characteristic time). On the other hand in the final part of the decaying phase only the damping is present $\left(f \sim \exp \left(-t / t_{\mathrm{d}}\right)\right)$. Summarizing these facts we can expect only the same trends of the characteristic times $t_{\mathrm{a}}$ and $t_{\mathrm{d}}$, which seems to be in agreement with our results.

According to the detailed study, in most cases of the selected spikes a plateau, indicating a trend of exponential type, was recognized in the decaying parts of spikes. However, there are some cases in which a plateau was also found in the ascending part. The $\tau$ was determined in a specific short interval only, when the interval with ideal monotonic connection among points of the spike profile was present. These differences in methods and intervals produced the differences in the resulting times $t_{\mathrm{a}}, t_{\mathrm{d}}$ and $\tau$. For the plasma temperature estimation, we used the derived values for $\tau$, since $\tau$ is determined by much higher precision than $t_{\mathrm{d}}$.

\section{c) Spike duration, polarization and time delays}

It is worth mentioning that the durations we found show a small range of uncertainty. Something similar is reported for the June 15, 1991 spikes at lower frequencies (Zlobec \& Karlický 1998).

For spikes at $1420 \mathrm{MHz}$ the duration (at half power) fits well the value obtained using the formula by Güdel \& Benz (1990), i.e. $9.5 \pm 1.0 \mathrm{~ms}$, which was derived for spikes up to about $1 \mathrm{GHz}$. For the spikes of March 28, 2001 at $2695 \mathrm{MHz}$ the initial mean values (during $16 \mathrm{~s}$ ) were consistent with the Güdel \& Benz (1990) formula, i.e. $4.0 \pm 0.7 \mathrm{~ms}$, whereas $3 \mathrm{~min}$ later they slightly exceeded this limit as they were in the range 5.0-5.8 ms. The other spikes of the same day and of June 6, 2000 showed a duration that was about $5.0 \mathrm{~ms}$ or longer.

The polarization of spikes at $1420 \mathrm{MHz}$ were rather constant and essentially almost zero. At $2695 \mathrm{MHz}$ the polarization degree was generally about $70 \% \mathrm{R}$, however also a group with variable values was detected. It is important to note that duration and polarization are independent parameters. Evident delays between the two polarimetric components were not detected.

For type I and type III bursts we know that they show higher polarization near the disk center than near the limb. When their polarization is lower they are also longer lasting, i.e. the scattering that performs the depolarization also prolongs the duration. That scattering region is far from the source region (Wentzel et al. 1986). For spikes it is different: the associated sources for the spikes here considered are relatively near the disk center and the polarization changes neither affect the duration nor the delay of one polarimetric component in respect to the other. These arguments point to the fact that the polarization should be originated in the source itself or near it.

We did not find a systematic time delay between the $\mathrm{R}$ and $\mathrm{L}$ components of the spike radiation. When it was present, it was generally in the range $0.1-0.3 \mathrm{~ms}$. For comparison, Benz \& Pianezzi (1997) found a systematic time delay $0.1 \mathrm{~ms}$. Due to the chaotic change of the sign of the time delay we explain that result in the same way as in the paper by Zlobec \& Karlický (1998), i.e. we assume different propagation trajectories of the $\mathrm{R}$ and $\mathrm{L}$ components in the turbulent source of the spikes.

\section{d) Nonlinear analysis}

For none of the analyzed time series any indication for the presence of a low-dimensional determinism was found, in agreement with previous studies. However, in 12 out of 16 subsequences indications for nonlinearity was found. Schwarz et al. (1993) presented evidence that the simultaneous appearance of spikes at different frequencies might be caused by a nonlinear process. From the present analysis we can infer that the temporal appearance of subsequent spikes at a fixed frequency is presumably not governed by a linear stochastic process but contains nonlinear signatures. The underlying dynamical system may be nonlinear stochastic or high-dimensional deterministic; low-dimensional determinism is most unlikely.

All models of spikes are based on some kind of plasma instability which can be expressed by the differential equation as follows

$\frac{\mathrm{d} w}{\mathrm{~d} t}=\gamma w$

where $t$ is time, $w$ is the wave energy density and $\gamma$ is the instability growth rate. Because the growth rate depends generally on the wave energy density $\gamma \equiv \gamma(w)$ the previous differential equation is nonlinear. Therefore, in this sense all models of spikes are implicitly nonlinear. The low number of equations in the most models of spikes is a result of a simplification of reality, and known stochastic aspects of spikes are usually not included into the quantitative modelling. That is why it is not easy to compare the present statistical results with most theoretical models. Nevertheless, there is the model of spikes proposed by Bárta \& Karlický (2001) that explicitly takes into consideration the nonlinearity and high-dimensionality by quantitative modelling of spikes as radio emission from the turbulent plasma in the flare reconnection outflows. Namely, the plasma turbulence represents a highly nonlinear system with a high (even in some cases infinite) dimensionality. Therefore, the model by Bárta \& Karlický (2001) agrees with the present statistical results.

Acknowledgements. H.M. and M.K. acknowledge the support from the grant IAA3003202 of the Academy of Sciences of the Czech Republic. H.M. wishes to express her gratitude for the kind hospitality and the financial support of the Institute of Astronomy, University of Graz, Austria. A.V. acknowledges the Austrian Fonds zur Förderung der wissenschaftlichen Forschung (FWF grant P15344) for supporting this project. P.Z. acknowledges the support given by MIUR (Italian Ministry for University and Research) in the frame of COFIN 2000. We are grateful to J. Magdalenić for preparing the list of the associated $\mathrm{X}$-ray flares.

\section{References}

Aschwanden, M. J. 1990, A\&AS, 85, 1141

Bárta, M., \& Karlický, M. 2001, A\&A, 379, 1045

Benz, A. O. 1986, Sol. Phys., 104, 99 
Benz, A. O., \& Pianezzi, P. 1997, A\&A, 323, 250

Benz, A. O., Zlobec, P., \& Jaeggi, M. 1982, A\&A, 109, 305

Diks, C., van Houwelingen, J. C., Takens, F., et al. 1995, Phys. Lett. A, 201, 221

Fleishman, G. D., \& Yastrebov, S. G. 1994, Sol. Phys., 154, 361

Fleishman, G. D., \& Melnikov, V. F. 1998, Physics - Uspekhi, 41, 1157

Fraser, A. M., \& Swinney, H. L. 1986, Phys. Rev. A, 33, 1134

Grassberger, P., \& Procaccia, I. 1983a, Phys. Rev. Lett., 50, 346

Grassberger, P., \& Procaccia, I. 1983b, Physica D, 9, 189

Grassberger, P., Schreiber, T., \& Schaffrath, C. 1991, Int. J. Bifurcation and Chaos, 1, 521

Güdel, M. 1990, A\&A, 239, L1

Güdel, M., \& Benz, A. O. 1990, A\&A, 231, 202

Güdel, M., \& Zlobec, P. 1991, A\&A, 245, 299

Hegger, R., Kantz, H., \& Schreiber, T. 1999, Chaos, 9, 413

Holman, G. D., Eichler, D., \& Kundu, M. R. 1980, in Radio Physics of the Sun, ed. M. R. Kundu, \& T. E. Gergeley, IAU Symp., 86, 465

Isliker, H. 1992, Sol. Phys., 141, 325

Isliker, H., \& Benz, A. O. 1994, A\&A, 285, 663

Isliker, H., \& Benz, A. O. 2001, A\&A, 375, 1040

Karlický, M. 1984, Sol Phys., 92, 329

Karlický, M., Sobotka, M., \& Jiřička, K. 1996, Sol. Phys., 168, 375

Krucker, S., \& Benz, A. O. 1994, A\&A, 285, 1038

Kuijpers, J., Van der Post, P., \& Slottje, C. 1981, A\&A, 103, 331

Melrose, D. B., \& Dulk, G. A. 1982, ApJ, 259, 844

Mészárosová, H., Karlický, M., Veronig, A., et al. 2000, A\&A, 360, 1126
Schreiber, T., \& Kantz, H. 1995, Chaos, 5, 143

Schreiber, T., \& Schmitz, A. 1996, Phys. Rev. Lett., 77, 635

Schreiber, T., \& Schmitz, A. 1997, Phys. Rev. E, 55, 5443

Schreiber, T., \& Schmitz, A. 2000, Physica D, 142, 346

Schwarz, U., Benz, A. O., Kurths, J., et al. 1993, A\&A, 277, 215

Slottje, C. 1981, Atlas of Fine Structures of Dynamic Spectra of Solar Type IV-dm and Some Type II Radio Bursts, Dwingeloo Observatory

Stähli, M., \& Benz, A. O. 1987, A\&A, 175, 271

Tajima, T., Benz, A. O., Thaker, M., et al. 1990, ApJ, 353, 666

Takens, F. 1981, in Dynamical Systems and Turbulence, ed. D. A. Rand, \& L. S. Young, Lecture Notes in Mathematics, 898 (Springer-Verlag), 366

Takens, F. 1985, in Dynamical Systems and Bifurcations, ed. B. L. J. Braaksma, H. W. Broer, \& F. Takens, Lecture Notes in Math., 1125 (Springer-Verlag), 99

Theiler, J. 1986, Phys. Rev. A, 34, 2427

Theiler, J. 1988, Phys. Lett. A, 135, 195

Theiler, J., Eubank, S., Longtin, A., et al. 1992, Physica D, 58, 77

Veronig, A., Messerotti, M., \& Hanslmeier, A. 2000, A\&A, 357, 337

Vlahos, L., \& Sharma, R. R. 1984, ApJ, 290, 347

Wang, S. J., Yan, Y.H., \& Fu, Q. J. 2002, Sol. Phys., 209, 185

Winglee, R. R., Dulk, G. A., \& Pritchett, P. L. 1988, ApJ, 328, 809

Wentzel, D. G. 1991, ApJ, 373, 285

Wentzel, D., Zlobec, P., \& Messerotti, M. 1986, A\&A, 159, 40

Zlobec, P., \& Karlický, M. 1998, Sol. Phys., 182, 477 